\title{
Ureteral Obstruction Caused by Endometriosis
}

\begin{tabular}{|l|l|l|}
\hline K. & Kaei & Nasu $^{\mathrm{a}}$ \\
\hline H. & Hisashi & Narahara $^{\mathrm{a}}$ \\
\hline T. & Takashi & Hayat $^{\mathrm{a}}$ \\
\hline I. & Isao & Miyakawa $^{\mathrm{a}}$ \\
\hline S. & Shin' ichi & Takahashi $^{\mathrm{b}}$ \\
\hline Y. & Yoshikazu & Fukunaga $^{\mathrm{b}}$ \\
\hline Y. & Yoshio & Nomura $^{\mathrm{b}}$ \\
\hline
\end{tabular}

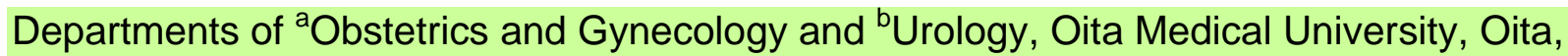
Japan

\section{Key Words}

Computerized tomography

Endometriosis

Hydronephrosis

Hydrometer

\section{Abstract}

The diagnosis of ureteral obstruction caused by endometriosis can be difficult. We present a 51-year-old multiparous Japanese woman who had undergone right salpingo-oophorectomy at the age of 19 years. She underwent total hysterectomy, left salpingo-oophorectomy, and adhesiotomy of the right ureter after the diagnosis of myoma uteri and endometriosis was made. Microscopic examination of the surrounding resected tissue confirmed the typical endometriotic origin. In addition to intravenous and retrograde pyelography, computerized tomography was useful to establish the diagnosis of ureteral obstruction caused by an extrinsic mass of endometriotic tissue.

Kaei Nasu, MD, PhD, Department of Obstetrics and Gynecology, Oita Medical University, Hasama-machi Oita 879-55 (Japan)

Introduction

An estimated proportion of 10-15\% of menstruating women suffer from endometriosis [1].

Urinary tract involvement occurs in approximately $1 \%$ of these cases, with a ratio of 40:5:1 affecting bladder, ureter, and kidneys, respectively [2, 3]. More than 100 cases of ureteral obstruction resulting from endometriosis have been published. We describe a case of ureteral endometriosis in whom urographic and computerized tomographic (CT) findings of ureteral obstruction proved useful in preopera-tive diagnosis.

\section{Case Report}

A 51-year-old Japanese woman, gravida 2, para 2, was admitted complaining of lumbago, dysmenorrhea, and hypermenorrhea. She had a history of a right salpingo-oophorectomy at the age of 19 years. Her blood pressure was 170/80 mm Hg. Pelvic examination revealed a uterine myoma about the size of a fist that was firm and tender on palpation.

Blood urea nitrogen and creatinine concentrations were 22.3 and $0.89 \mathrm{mg} / \mathrm{dl}$, respectively, and the CA-125, CA-72-4, and CA-19-9 levels were 74, 1.6, and $10.6 \mathrm{U} / \mathrm{ml}$, respectively. Other routine laboratory parameters were within normal limits. A Papanicolaou test was negative. 
Fig. 1. The CT scan confirms the presence of soft-tissue density around the distal right ureter that extended to the corpus uteri, suggestive of extrinsic compression caused by endometriosis at the point of obstruction.

An intravenous pyelogram demonstrated right moderate hydronephrosis and hydroureter due to an obstruction of the distal ureter. Ultrasonography showed moderate hydronephrosis and several myo-matous nodules (maximum diameter 14-38 mm) in the corpus uteri. A CT scan confirmed the presence of myomas in corpus uteri and connective tissue, surrounding the right distal ureter (fig. 1). These

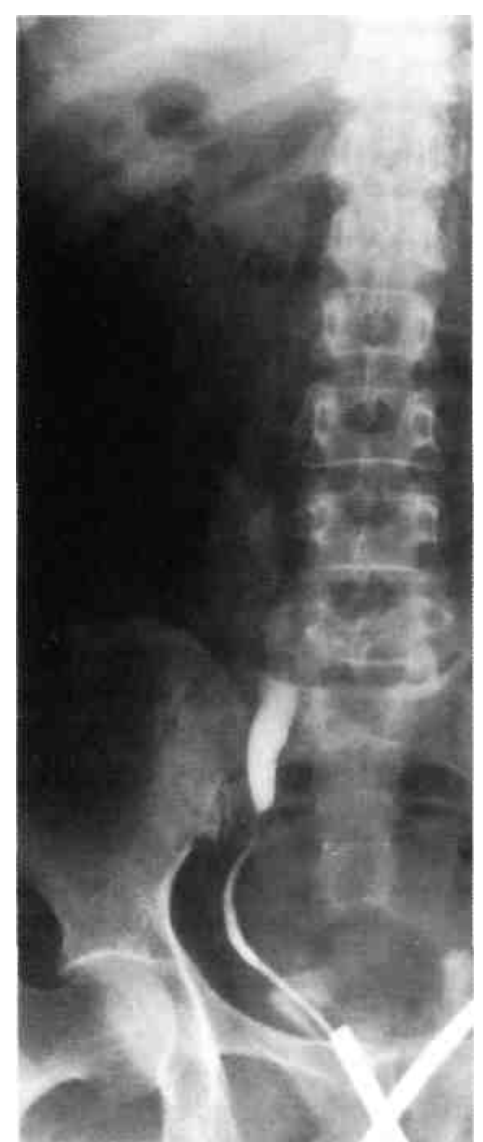

Fig. 2. A retrograde pyelogram demonstrated a moderate hy-droureter due to stenosis of the distal ureter. Mucosa and proximal and distal segments appear normal.

findings suggested extrinsic compression at the point of distal ureteral obstruction. Retrograde pyelography was performed, and a dou-ble-J catheter was inserted preoperatively (fig. 2)

Firm adhesion of intestine, left adnexa, corpus uteri, and distal ureter as well as several blueberry like spots on the pelvic peritoneum, were observed intraoperatively. A palpable thickening was noted within the wall of the narrowed segment of the ureter. We performed total hysterectomy, left salpingo-oophorectomy, and adhesiotomy of the right ureter. Microscopic evaluation of the resected tissue surrounding the obstruction revealed typical endometriotic tissue. Hormonal therapy was not nesessary. The patient did well, and an intravenous pyelogram performed 2 months postoperatively demonstrated free flow of contrast medium to the bladder.

Discussion 
Ureteral obstruction is a rare complication of endometriosis, and involvement of the urinary tract occurs in approximately $1 \%$ of such cases [2]. Several studies reported a high incidence of prior pelvic surgery (60-70\%) in patients with endometriosis in the urinary tract [3], consistent with our case.

On physical examination, patients with ureteral obstruction caused by endometriosis show nonspecific symptoms and findings. It is, therefore, important to rule out ureteral involvement in patients with endometriosis. Ultrasonographic and radiologic examinations are necessary for the early detection of silent obstructive uropathy in such patients.

Pollack and Wills [4] reviewed the urographic findings of ureteral endometriosis in patients whose radiologic findings were nonspecific and observed hydrouretero-nephrosis, narrowing of the pelvic ureter, normal appearance of the ureter distal to the diseased segment, and, rarely, the presence of an intraluminal ureteral mass. The degree of obstructive uropathy was often severe. Ureteral strictures are characteristically smooth, often tapering abruptly without any 'shoulder' effect.

The intravenous pyelogram in our case was a valuable indicator of the severity of the obstruction, its approximate location, and the laterality of the involvement. It also provided a means of comparison for posttreatment studies. Retrograde pyelography was useful for evaluating precise location, extent, configuration, and degree of stenosis as well as mucosal appearance. Insertion of a double-J catheter also aided us in the treatment of this patient. Pious et al. [5] described the CT scan findings of ureteral endometriosis. These authors reported a case whose CT scan showed soft-tissue density around the distal right ureter, suggestive of extrinsic compression at the point of obstruction. The radiographic findings in our case were similar to those described in previous studies.

In addition to the intravenous and the retroverted pyelograms, the CT scan proved useful for the diagnosis of ureteral obstruction caused by an extrinsic mass of endometriotic tissue.

References 1 Metzger DA, Haney AF: Endometriosis: Etiology and pathophysiology of infertility. Clin Ob-stetGynecol 1988;31:801-812.

Yates-Bell AJ, Molland EA, Chir B, Pryor JP: Endometriosis of the ureter. Br J Urol 1972;44: 58-67.

Stillwell TJ, Kramer SA, Lee RA: Endometriosis of ureter, Urology 1986;28:81-85.

Pollack HM, Wills JS: Radiographic features of ureteral endometriosis. Am J Roentgenol 1978; 131:627-631.

Pious RH, Sunshine R, Goldman H, Schwartz IS: Ureteral endometriosis in post-menopausal women. Urology 1985;26:408-411.

\section{6}

Nasu/Narahara/Hayata/Miyakawa/ Ureteral Obstruction Caused by

Takahashi/Fukunaga/NomuraEndometriosis 